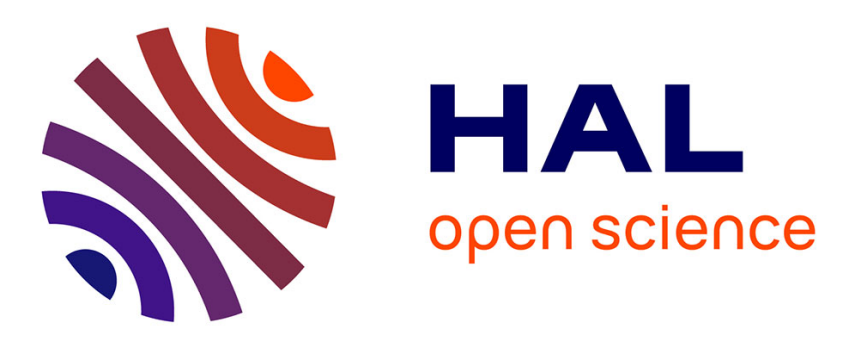

\title{
Snapshot imaging of postpulse transient molecular alignment revivals
}

\author{
V. Loriot, R. Tehini, E. Hertz, B. Lavorel, O. Faucher
}

\section{To cite this version:}

V. Loriot, R. Tehini, E. Hertz, B. Lavorel, O. Faucher. Snapshot imaging of postpulse transient molecular alignment revivals. Physical Review A : Atomic, molecular, and optical physics [1990-2015], 2008, 78, pp.013412. hal-00397236

\section{HAL Id: hal-00397236 https://hal.science/hal-00397236}

Submitted on 19 Jun 2009

HAL is a multi-disciplinary open access archive for the deposit and dissemination of scientific research documents, whether they are published or not. The documents may come from teaching and research institutions in France or abroad, or from public or private research centers.
L'archive ouverte pluridisciplinaire HAL, est destinée au dépôt et à la diffusion de documents scientifiques de niveau recherche, publiés ou non, émanant des établissements d'enseignement et de recherche français ou étrangers, des laboratoires publics ou privés. 


\title{
Snapshot imaging of postpulse transient molecular alignment revivals
}

\author{
V. Loriot, R. Tehini, E. Hertz, B. Lavorel, and O. Faucher \\ Institut Carnot de Bourgogne, UMR 5209 CNRS-Université de Bourgogne, \\ 9 Avenue A. Savary, BP 47 870, F-21078 Dijon Cedex, France
}

\begin{abstract}
Laser induced field-free alignment of linear molecules is investigated by using a single-shot spatial imaging technique. The measurements are achieved by femtosecond time-resolved optical polarigraphy (FTOP). Individual alignment revivals recorded at high resolution in $\mathrm{CO}_{2}$, as well as simultaneous observation of several alignment revivals produced within the rotational period of the $\mathrm{O}_{2}$ molecule are reported. The data are analyzed with a theoretical model describing the alignment experienced by each molecule standing within the interaction region observed by the detector. The temporal dynamics, intensity dependence, and degree of alignment are measured and compared with the awaited results. The technique is simple and can be easily implemented in a large class of molecular samples. Improvement to extend the performance of the method is discussed. The reported study is a decisive step towards feedback optimization and optimal control of field-free molecular alignment.
\end{abstract}

PACS numbers: $37.10 . \mathrm{Vz}, 42.50 . \mathrm{Hz}, 42.50 . \mathrm{Md}$

\section{INTRODUCTION}

It is well known that a short laser pulse (of duration $\tau_{\mathrm{p}} \ll T_{\mathrm{r}}$, with $T_{\mathrm{r}}=1 /(2 B c)$ the rotational period, $B$ the rotational constant, and $c$ the speed of light) yields postpulse transient molecular alignment revivals $[1,2]$. This effect called non-adiabatic or field-free alignment has been studied extensively, both using linearly [3-5], elliptically [6], and cross polarized lights $[7,8]$. Elegant strategies to optimize the degree of alignment have been proposed, either based on the use of a sequence of Fourier transform limited pulses $[9,10]$, or on tailoring a single laser pulse $[11,12]$. Among them, some have been experimentally implemented to enhance molecular field-free alignment $[13,14]$. The techniques that have been employed so far to measure molecular alignment can be cast into two categories. One way is to break a previously aligned molecule and to observe the angular distribution of the resulting photofragments [15]. The combination with a two-dimensional imaging detection makes this technique very powerful; it allows under precautionary conditions the reconstruction of the angular distribution of the precursor molecule and therefore a visualization of the aligned molecule. The second category rests upon the modification of the optical properties of the medium when bulk molecules are aligned. It can be explained in terms of optical Kerr effect (OKE) produced by the reorientation of the molecules imposed by the external field. The degree of alignment at a certain time can be measured by observing a probe pulse after propagation through the sample of aligned molecules. It has been shown that the change of polarization [16] and spatial profile $[6,17]$ of the probe is directly related to the director cosines used to describe the orientation of the molecular axes in the laboratory frame, from which the alignment can be inferred.

In order to observe the temporal dynamics of fieldfree alignment (occurring typically in the picosecond time scale), both above mentioned techniques require to re- peat the measurements by tuning the time delay between the aligning and probe pulse. Additionally, whereas the OKE technique allows in principle single-shot measurements, the photofragments imaging needs an averaging of the data over a large number of laser shots. So far, to our knowledge, only three experiments of single-shot measurements of transient molecular alignment revivals have been reported [18-20]. All are based on OKE. In Ref. [18], a weak field molecular alignment produced by an unfocused laser beam is detected by a chirped probe pulse with a birefringence technique. Knowing the chirp parameter, the signal measured in the frequency domain can be converted into the time domain. In Ref. [19], the authors make use of spectral interferometry to reconstruct the phase of a chirped probe pulse co-propagating with the aligning pulse. Using a space sensitive detection, they can retrieve through the change of the refractive index the alignment produced over the transverse profile of the pulse. Very recently, spectral interferometry has also been used for single-shot measurement of the phase modulation produced by a revival of transient alignment [20]. We note that in all cited works, the temporal window of the detection is limited by the stretching of the laser pulse (500 fs, $2 \mathrm{ps}$, and $1.3 \mathrm{ps}$ in [18], [19], and [20], respectively).

Another ultrafast imaging technique based on OKE is the femtosecond time-resolved optical polarigraphy (FTOP) [21]. Developed for the purpose of visualizing the instantaneous intensity distribution of light, it was initially applied to the propagation of a femtosecond laser pulse in absorbing less medium [22]. However, it has also been envisaged as a possibility to resolve rotational recurrences produced by the interaction of a short pulse with a molecular sample [23].

The scope of this work is to report on high resolution, space- and time-sensitive measurements of field-free molecular alignment, achieved on a large temporal window (up to $18 \mathrm{ps}$ ) using the single-shot FTOP technique. In particular, we will show that an FTOP image not only 
carries the information about the intensity dependence and the temporal dynamics of the alignment, but also allows a precise estimation of the laser intensity in the molecular sample. The principle of the FTOP method and the experimental setup will be presented in section II, followed by the theoretical model in section III. Section IV will be dedicated to the presentation and analysis of the alignment signals observed in $\mathrm{CO}_{2}$ and $\mathrm{O}_{2}$. A conclusion with a comparison to other works will be given in section $\mathrm{V}$.

\section{PRINCIPLE AND METHOD}

The principle of the experiment can be described as follows. A first laser pulse (pump pulse), linearly polarized, is used to produce a non-resonant excitation of the molecules present in a gas cell. The amplitude of the induced dipole, which depends on the intensity of the incident field, causes a change of the refractive index of the medium through OKE. When considering molecules of spherical symmetry (for instance, $\mathrm{CH}_{4}$ in its lower vibrational state) the induced dipole results solely from the deformation of the electronic cloud, like with atoms. At the time scale of a standard femtosecond laser, the response of the medium can be regarded as sudden. However, most of the molecules shows an anisotropy of polarizability. For these molecules, the magnitude of the induced dipole not only depends on the field strength but also on its orientation with respect to the field. Since only $\mathrm{CO}_{2}$ and $\mathrm{O}_{2}$ will be considered in this work, from now on we will focus our discussion on linear molecules. In order to satisfy to the principle of minimum energy, the result of the interaction between the induced dipole and the field is a rotation of the molecular axis towards the direction of the field, leading to the alignment of the molecule in the space-fixed frame. In the non-adiabatic regime, the alignment occurs through recurrences produced after the pulse turn-off. However, for not too heavy molecules like $\mathrm{CO}_{2}$ and $\mathrm{O}_{2}$, the effect of alignment is already significant during the pulse, as it will be shown in section IV. In order to measure the alignment initiated by the pump pulse, we use a second pulse (probe pulse) of linear polarization and weaker energy, time-delayed with respect to the pump pulse. The role of this probe is to detect the change of the refractive index resulting from the molecular alignment. Because both pulses are linearly polarized along different directions, the anisotropy of the alignment results in a birefringence signal detected by the probe. Several works have benefited of this effect to measure the alignment of linear $[4,18,24-26]$ and asymmetric $[16,26]$ top molecules when using quasi co-propagative beams.

The setup of the experiment is depicted in Fig. 1. The pump and probe beams are produced by a chirped pulse amplified Ti:sapphire femtosecond laser. The system provides 100 fs-duration pulses, with a wavelength centered at $800 \mathrm{~nm}$, and with $1 \mathrm{kHz}$ repetition rate. The molecules are contained in a $30-\mathrm{cm}-$ long static cell (GC) at room

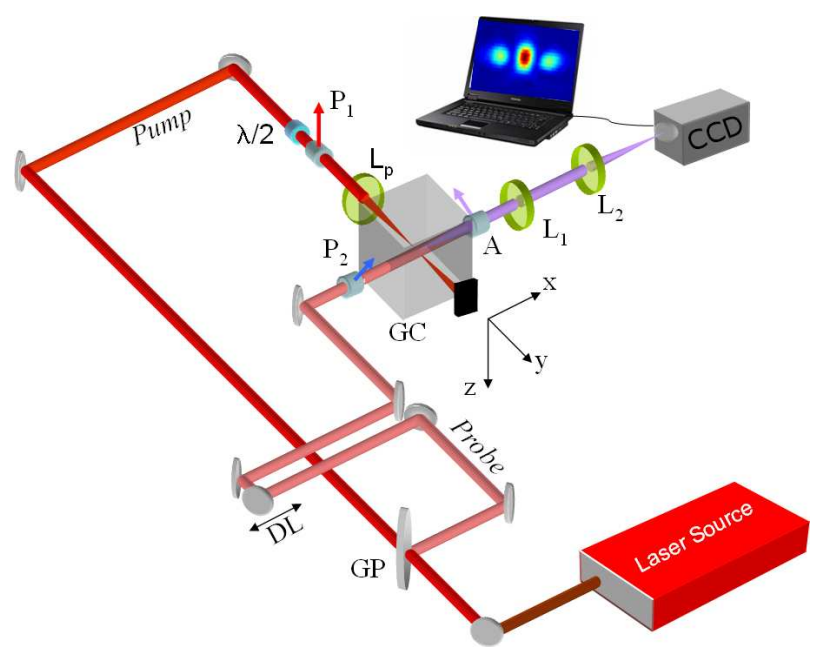

FIG. 1: (Color online) Experimental setup for the FTOP measurements. L: lenses, P: polarizers, A: analyzer, GP: Glass plate, DL: delay line, GC: gas cell, CCD: camera.

temperature. In the experiment performed with $\mathrm{CO}_{2}$ $\left(\mathrm{O}_{2}\right)$, the pump beam is focused along the $y$ axis with a lens $\left(\mathrm{L}_{\mathrm{P}}\right)$ of $20 \mathrm{~cm}(45 \mathrm{~cm})$ focal length. A half-wave plate $(\lambda / 2)$ combined with a polarizer $\left(\mathrm{P}_{1}\right)$ is used to adjust the energy and to define the polarization of the field along the $z$ axis. The probe pulse, of weaker energy, is produced through a reflection of the pump beam on a glass plate (GP). After passing through a polarizer $\left(\mathrm{P}_{2}\right)$ that sets its field vector at $45^{\circ}$ with respect to the $z$ axis in the $y z$ plane, the collimated beam enters the cell, intersects at a right angle the pump beam, exits the cell, and then crosses a second polarizer, i.e. the analyzer (A), rotated by $90^{\circ}$ with respect to the first one. Because the refractive index seen by the probe is different along the $y$ and $z$ directions, the original polarization of the probe becomes slightly elliptical after its interaction with the aligned molecules. The amount of probe energy that passes through the analyzer is therefore related to the alignment initiated by the pump pulse. An imaging system made of two lenses $\left(\mathrm{L}_{1}\right.$ and $\left.\mathrm{L}_{2}\right)$ is used to image the overlapping region of the two beams onto the chip of a charge-coupled device (CCD). The magnification factor $M$ of the imager is different depending on the investigated molecules: $M=-2.11$ and -1.06 for $\mathrm{CO}_{2}$ and $\mathrm{O}_{2}$, respectively. In both cases, the depth of focus $d_{f} \approx 500 \mu \mathrm{m}$ is much larger than the size of the pump beam at focus. The time delay between the two pulses can be adjusted with a delay line (DL) inserted on the probe beam path.

With the setup of Fig. 1, the image measured by the CCD camera can be seen as a projection of a portion of the pump beam pathway on the detector plane $(y z)$. Because of the cross beam geometry, the time axis is converted by the probe along the space axis $y$ of the image (the propagation direction of the pump beam), whereas the $z$ axis reflects the intensity distribution of the pump beam along this direction. By adjusting the size of the 
collimated probe beam and the delay between the two pulses, the temporal window of the measurements can be adjusted in width and position. The technique allows therefore to observe snapshots of individual or several space-resolved transient molecular alignment revivals. In all measurements, the confocal parameter $b$ (defined as two times the Rayleigh distance) of the pump beam is larger than the region observed by the CCD camera.

\section{NUMERICAL MODEL}

The pump and probe electric fields are chosen to have both a spatial and temporal Gaussian form of same duration $\tau_{\mathrm{p}}$. For simplification, we assume that the propagation through the gas sample occurs at the group velocity $v_{\mathrm{g}} \simeq c$. The origin of space $x=y=z=0$ is defined at the crossing point of the two beam axes. The intensity of the pump beam propagating along $y$ writes as

$$
\begin{aligned}
I_{\mathrm{p}}(x, y, z, t)= & I_{0 \mathrm{p}}\left(\frac{w_{0}}{w(y)}\right)^{2} \exp \left(-\frac{2\left(x^{2}+z^{2}\right)}{w^{2}(y)}\right) \\
& \times \exp \left(-4 \ln 2\left(\frac{t-y / c}{\tau_{\mathrm{p}}}\right)^{2}\right),
\end{aligned}
$$

where $w(y)$ is the beam radius, $w_{0}$ is the beam waist, and $I_{0_{\mathrm{p}}}$ is the peak intensity. The alignment of the molecular sample produced through the pump interaction results in a space- and time-dependent transient birefringence $\Delta n=n_{z}-n_{y}[25]$ :

$$
\Delta n(x, y, z, t)=\frac{3 N}{4 n \epsilon_{0}}\left[\Delta \alpha\left(\left\langle\cos ^{2} \theta\right\rangle(t)_{\psi_{x, y, z}}-\frac{1}{3}\right)\right],
$$

with $n_{z}$ and $n_{y}$ the refractive index respectively parallel and perpendicular to the pump polarization direction, $n$ the average value of the refractive index at the probe frequency, $N$ the number density, and $\epsilon_{0}$ the dielectric constant of vacuum. The alignment term $\left\langle\cos ^{2} \theta\right\rangle(t)_{\psi_{x, y, z}}$ refers to the expectation value of $\cos ^{2} \theta$, with $\theta$ the angle between the molecular axis and the polarization direction of the pump electric field. The state vector $\psi_{x, y, z}(t)$ can be evaluated at the space coordinate $(x, y, z)$ by solving the time dependent Schrödinger equation $i \hbar d \psi / d t=H \psi$ with

$$
H=H_{\text {rot }}-\frac{1}{4} \alpha_{z z} I_{\mathrm{p}}(x, y, z, t)
$$

the effective Hamiltonian that describes the non-resonant excitation of the molecular polarizability, $H_{\text {rot }}$ the rotational Hamiltonian (including the first-order correction for the centrifugal distortion), and $\alpha_{z z}$ the diagonal laboratory-fixed component of the polarizability with respect to the pump field direction. Higher-order polarizabilities (i.e., hyperpolarizabilities) can be neglected under the intensity regime investigated in this work. In order to account for finite temperature, $\left\langle\cos ^{2} \theta\right\rangle(t)_{\psi_{x, y, z}}$ must be averaged over the thermal distribution of the initial rotational states.
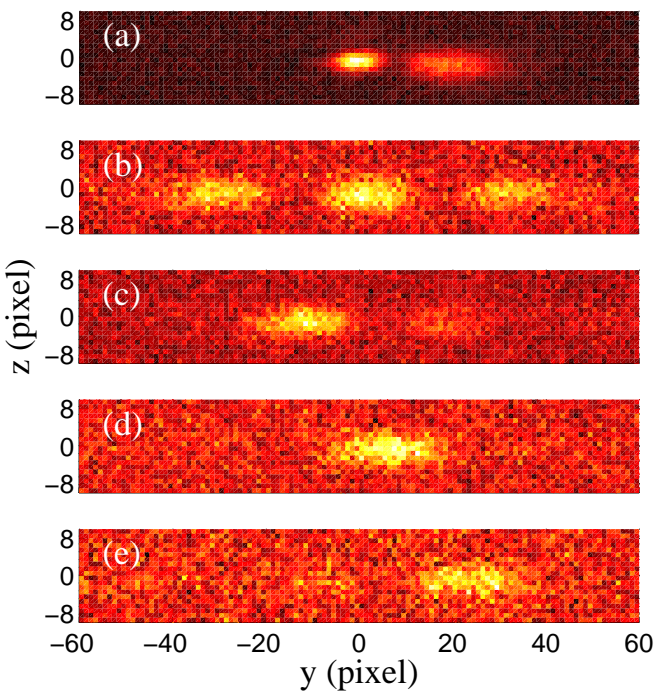

FIG. 2: (Color online) Single-shot images of transient molecular alignment revivals of $\mathrm{CO}_{2}$ at room temperature. The energy of the aligning pulse is $40 \mu \mathrm{J}$. The pump-probe delay $\tau$ is adjusted to be a multiple of the quarter rotational period: 0 (a), $T_{\mathrm{r}} / 4$ (b), $T_{\mathrm{r}} / 2$ (c), $3 T_{\mathrm{r}} / 4$ (d), and $T_{\mathrm{r}}(\mathrm{e})$.

The image captured by the CCD camera is related to the birefringence $\Delta n$ and the field amplitude $E_{\mathrm{pr}}$ of the delayed probe pulse propagating along $x$ [21]:

$$
\begin{aligned}
\mathcal{S}(y, z, \tau) \propto & \int_{-\infty}^{\infty} d t\left\{\int_{-\infty}^{\infty} d x E_{\mathrm{pr}}(x, y, z, t-\tau-x / c)\right. \\
& \times \Delta n(x, y, z, t)\}^{2}
\end{aligned}
$$

In this expression, we assume that (i) the birefringence effect experienced by the probe beam is weak, $\Delta n \ll$ $\left\{k \omega_{0}\right\}^{-1}$, with $k$ the wave vector and (ii) the intensity of the probe is low enough so that it does not produce any additional molecular alignment. The time interval $\tau+x / c$ accounts for the delay $\tau$ adjusted between the pump and probe pulse, as well as for an additional temporal shift $x / c$ introduced by the crossing of the probe beam over the transverse profile of the pump beam.

From the model, we see that $\mathcal{S}(y, z, \tau)$ comprises the alignment produced at different positions $y$ along the propagation of the pump that correspond to different times $t-y / c$ of creation [see Eq. 1]. The temporal evolution of the alignment is thus mapped into the spatial coordinate $y$ of the signal.

\section{RESULTS AND DISCUSSION}

The sensitivity of the FTOP technique permits to record single-shot, space- and time-resolved images of field-free molecular alignment. Figure 2 displays snapshots of $\mathrm{CO}_{2}$ recorded for different pump-probe delays. 

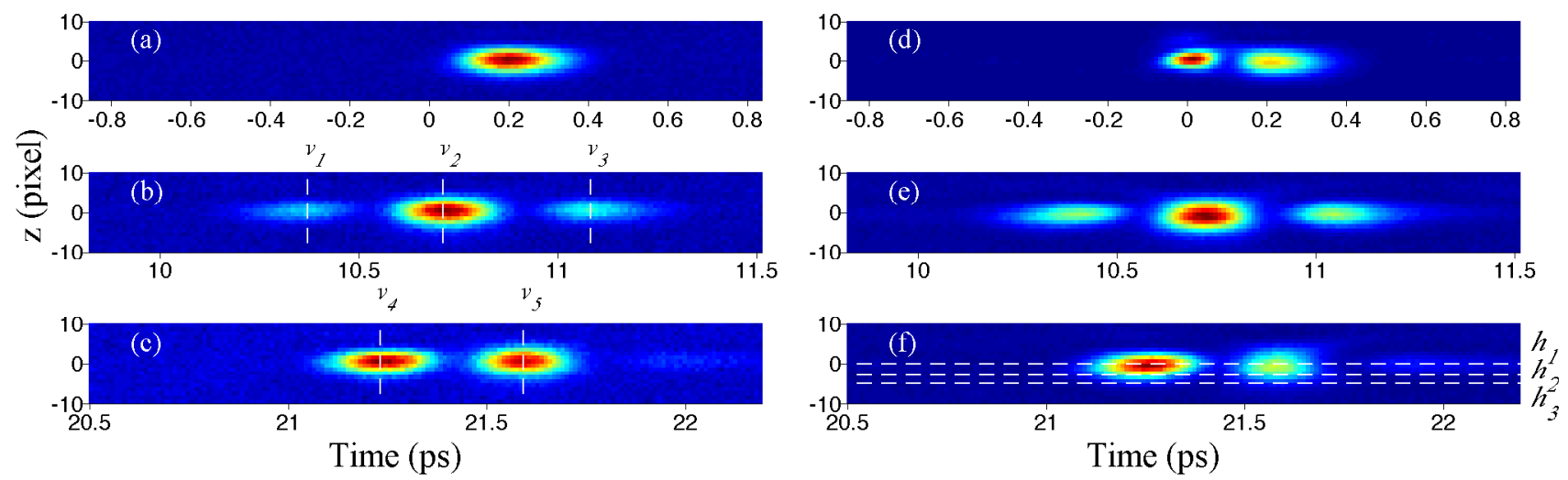

FIG. 3: (Color online) Averaged images of transient molecular alignment revivals of $\mathrm{CO}_{2}$ recorded for a pulse energy of 20 (a-c) and $40 \mu \mathrm{J}(\mathrm{d}-\mathrm{f})$. The delay $\tau$ between the two pulses is $0(\mathrm{a}, \mathrm{d}), 10.7(\mathrm{~b}, \mathrm{e})$, and $21.4 \mathrm{ps}(\mathrm{c}, \mathrm{f})$.

The experiment has been achieved using a collimated probe beam of 4-mm-diameter and a stagnation pressure of 1 bar. The ordinate $z$ of the figure is along the transverse radius of the pump beam (see Fig. 1). The abscissa $y$ corresponds to the propagation axis of the pump pulse. The axes are labeled in units of pixel of the CCD camera. During the propagation of the pump pulse, the interaction with the molecules occurs at different times with respect to their positions along the $y$ axis. In each $y z$ plane, the temporal response of the molecules excited by the pump is thus converted by the probe pulse into the spatial $y$ coordinates of the image. The time calibration of the imaging system was achieved by tuning the pumpprobe delay and measuring the resulting displacement of each single transient along the $y$ axis. Considering the pixel size of the CCD camera $(6.7 \mu \mathrm{m} \times 6.7 \mu \mathrm{m})$ and the magnification factor $M=-2.11$ of the imaging system, this displacement translates to a total range of $\approx 1.3 \mathrm{ps}$ for the measurements of Fig. 2. It is worth noticing that the time axes in Fig. 2 should be seen as oriented from left to right, in consistence with the pump pulse propagating along the positive $y$ axis (see Fig. 1) and the inversion produced by the imaging system of negative magnification.

The images in Figs. 2 (b-e) correspond respectively to the first, second, third, and fourth alignment revival of $\mathrm{CO}_{2}$ occurring within the rotational period $T_{\mathrm{r}} \approx 42.7 \mathrm{ps}$. The resolution of the imaging system is sufficient to distinguish the fine structure of each transient that it is a prerequisite for an accurate reconstruction of the alignment. The snapshot recorded around $\tau=0$ is shown in Fig. 2 (a). When the pump and probe overlap, the birefringence experienced by the probe beam results from the two contributions mentioned in section II, i.e. the prompt electronic and the retarded rotational response. At room temperature, the alignment peak of $\mathrm{CO}_{2}$ occurs after turn-off of the pulse. With the resolution of the present measurements it is hence possible to observe both effects. The electronic response is manifested in Fig. 2 (a) by the narrow structure located around pixel 0 . The retarded response produced by the molecular alignment is observed on the right side of the image, with a maximum located around pixel 20.

In order to proceed with a thorough analysis of the effects observed in Fig. 2, it is preferable to improve the signal-to-noise ratio by averaging the measurements over several laser shots. Although it fatally introduces a loss of information, this one is however limited by the relatively good stability of the laser system (2-3\% shot to shot variation in energy). FTOP images averaged over 100 laser shots are shown in Figs. 3 for two different energies of the aligning pulse. For convenience, the figures will be presented from now on with the abscissa axes converted in time, with the origin coinciding with the maximum of the electronic response [see Fig. 2 (a)]. The noticeable difference between the two columns is the alteration of the transient shapes. The comparison between Figs. 3 (b-c) and 3 (e-f) highlights distinct degrees of alignment. The images recorded at $\tau=0$ show an additional feature resulting from the combination between the electronic and rotational response. At low energy [Fig. 3 (a)], the rotational effect predominates and only one unstructured transient is observed. At higher energy [Fig. 3 (d)], this transient clearly separates into the two sub-structures already observed in Fig. 2 (a). We notice the larger amplitude of the electronic response (centered around $0 \mathrm{fs}$ ) compared with the rotational one (centered around $210 \mathrm{fs}$ ). The fact that the two contributions appear well separated, rather than through of a broad convoluted structure, is ascribed to an interference effect. In Fig. 3 (d) the electronic response occurs through a higher nonlinear process contributing to a birefringence of opposite sign compared to the rotational contribution [32]. The cancellation between the prompt and retarded responses leads to a minimum of signal observed around 105 fs.

To determine the degree of alignment produced in Figs. 3 , it is necessary to estimate as a preliminary the 
intensity to which the molecules are exposed during the experiment. The pulse duration is determined from autocorrelation measurements. In order to account for a possible alteration of the pump beam resulting from its propagation through the nonlinear medium, the transverse profile of the pump pulse is deduced from the FTOP images. For this purpose, we have reported in Fig. 4 the signal $\mathcal{S}(z)$ measured along the fives vertical lines shown in Figs. 3 (b) and (c). The curves displayed with full lines have been obtained by fitting these data with Eq. (4). In this procedure, we have assumed that the alignment contributes to the birefringence signal by a term proportional to the pump intensity: $\Delta n(x, y, z) \propto I_{\mathrm{p}}(x, y, z)$. This assumption is justified for the intensity and temperature investigated in Figs. 3 (b) and (c). The data are globally well reproduced using the Gaussian beam approximation of Eq. (1), albeit a small asymmetry with respect to the beam center that might be due to an aberration of the pump laser beam. Expressed in the image plane, the adjusted value for the beam waist is $\approx 4$ pixels, which corresponds to $\omega_{0} \approx 18 \mu \mathrm{m}$ in the real space. If we assumes a beam of cylindrical symmetry, the estimated peak intensity $I_{0 \mathrm{p}}$ [see Eq. (1)] for Figs. 3 (a-c) and Figs. 3 (d-f) is hence 37 and $74 \mathrm{TW} / \mathrm{cm}^{2}$, respectively.

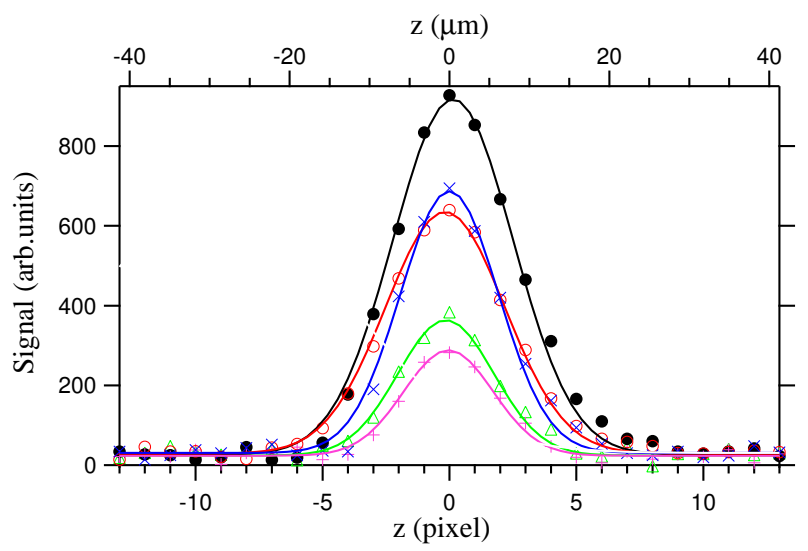

FIG. 4: (Color online) Signal distribution along the transverse direction $z$ of the pump beam. The different symbols refer to the vertical lines labeled $v_{1}(+), v_{2}(\bullet), v_{3}(\triangle), v_{4}(\circ)$, and $v_{5}(\times)$ in Figs. $3(\mathrm{~b})$ and $(\mathrm{c})$. Numerical fits of the transverse profile of the pump beam along the $z$ axis (full lines).

The advantage of the FTOP technique is that the time and intensity dependence of the degree of alignment can be simultaneously obtained starting from a single image. The signal measured along different horizontal lines ( $y$ axis) of the CCD camera reflects the alignment produced at different intensities within the pump beam profile. To highlight this aspect, we have reported in Fig. 5 the signal $\mathcal{S}(y)$ obtained from Fig. 3 (f) at the three different ordinates labeled $h_{j}(j=1,2,3)$. The comparison between the three graphs shows the alteration of the signal produced by the molecules located at different radial distances $|z|=5$ pixels $\left(h_{3}\right)$ and 3 pixels $\left(h_{2}\right)$ from the
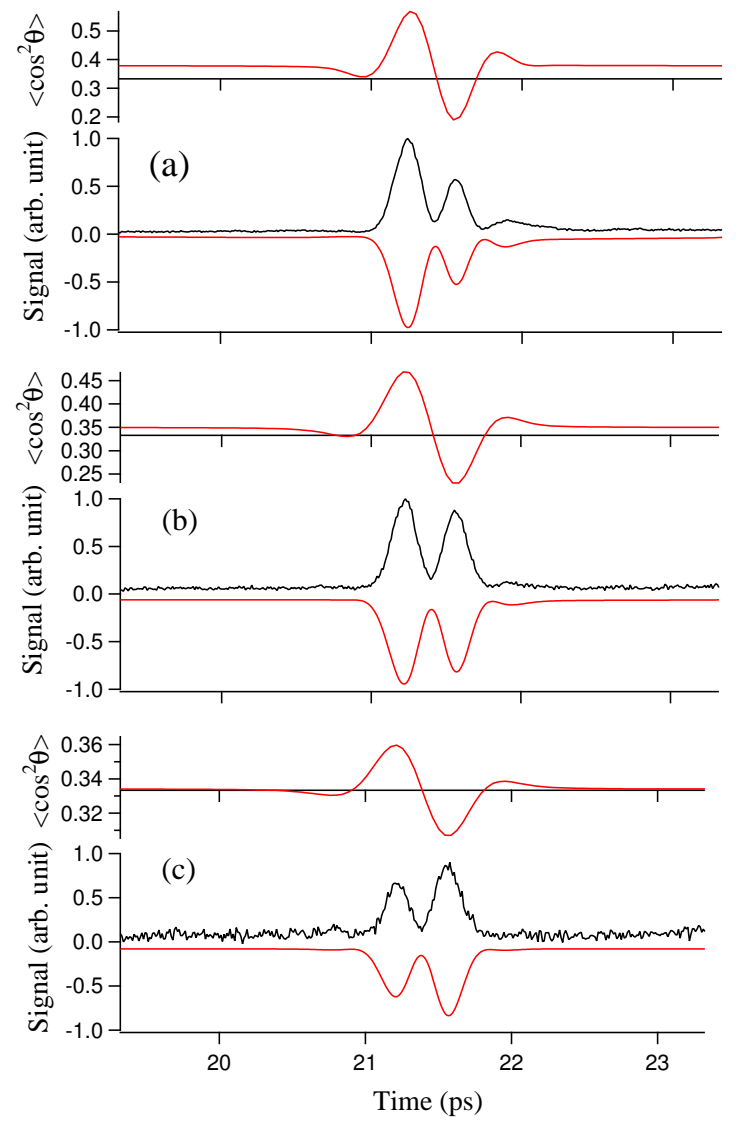

FIG. 5: (Color online) Lower graphs: signals measured along the propagation axis $y$ of the pump beam, deduced from the horizontal cuts labeled $h_{1}$ (a), $h_{2}$ (b), and $h_{3}$ (c) in Fig. 3 (f). The numerical simulations (shown reversed) have been obtained for a peak intensity $I_{0 \mathrm{p}}=62 \mathrm{TW} / \mathrm{cm}^{2}$. Upper graphs: $\left\langle\cos ^{2} \theta\right\rangle$ calculated for the molecules exposed at a pump intensity $I_{\mathrm{p}}=62(\mathrm{a}), 34(\mathrm{~b})$, and $7 \mathrm{TW} / \mathrm{cm}^{2}$ (c) (see text).

beam center $z=0$ pixel $\left(h_{1}\right)$. Based on the simulation of Eq. (4), the calculated signals have been obtained by adjusting the peak intensity $I_{0 \mathrm{p}}$ to $62 \mathrm{TW} / \mathrm{cm}^{2}$, a value that leads to the best agreement with the observation. This intensity is close to the intensities deduced from the measurement of the beam waist, i.e. $74 \mathrm{TW} / \mathrm{cm}^{2}$, which is quite satisfactory considering the assumption of a perfect Gaussian beam made in the model. It is worth mentioning that all the simulations presented in this work have been performed with a pulse duration corresponding to the measured values (100 fs). The expectation values $\left\langle\cos ^{2} \theta\right\rangle$ introduced in Eq. 2 are shown in the upper parts of the figures, on the scaled right axis of the graphs. At this point, we should recall that the depth of focus $d_{f}$ of the imaging system is larger than the spot of the pump beam. Each experimental data point presented in Fig. 5 results therefore from a one-dimensional spatial averaging of the molecular alignment produced along the propagation axis of the probe beam. The expectation values shown in Fig. 5 are those calculated for 


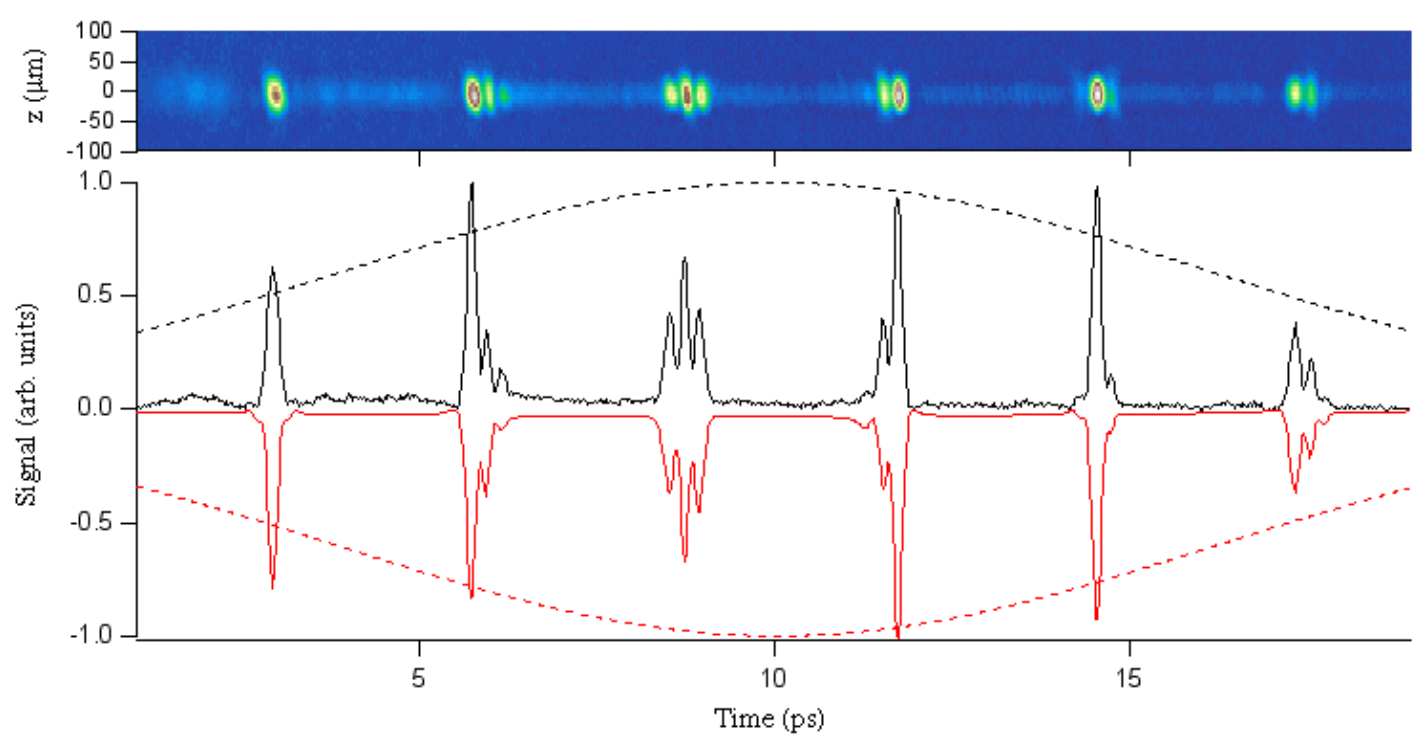

FIG. 6: (Color online) Upper graph: Averaged image (100 shots) recorded in $\mathrm{O}_{2}$ at room temperature and atmospheric pressure. The pump-probe delay was set at $\tau=10$ ps. Lower graph: signal (full line) extracted from the horizontal central line of the CCD image $(z=0)$. The theoretical signal (shown reversed) has been calculated at a peak intensity of $49 \mathrm{TW} / \mathrm{cm}^{2}$. Transverse profile of the probe beam (dashed lines).

the molecules located at $x=0, z=h_{j}(j=1,2,3)$ with the corresponding intensity $I_{\mathrm{p}}=62,34$, and $7 \mathrm{TW} / \mathrm{cm}^{2}$, respectively. The largest signal is observed in Fig. 5 (a), where molecules are exposed to the most intense part of the pump beam, including the peak intensity $I_{0 \mathrm{p}}$. The $\left\langle\cos ^{2} \theta\right\rangle$ value shown for $I_{\mathrm{p}}=I_{0 \mathrm{p}}=62 \mathrm{TW} / \mathrm{cm}^{2}$ is modulated between 0.57 , where the alignment of the molecules is maximum, and 0.19 , where the delocalization of the molecular axis in the plane perpendicular to the pump field is maximum. Before (after) the alignment (delocalization) transient, a small permanent alignment is produced, denoted by the presence of an elevated base line $\left\langle\cos ^{2} \theta\right\rangle \simeq 0.35>1 / 3$ [27]. For the molecules standing in the peripheral area of the beam center, Figs. 5 (b and c), and therefore exposed to lower intensities as compared to Fig. 5 (a), the contrast between alignment and planar delocalization is reduced together with the degree of permanent alignment. As shown in a previous work [25], the structural modification of the revival, in particular the asymmetry reversal observed between Fig. 5 (a) and (c), is a signature of strong field alignment.

It is possible to increase the temporal window of the detection to record at once several transients of molecular alignment. In the setup, the temporal range is limited by the aperture of the analyzer that restricts the beam size of the probe. In order to increase the detection window, the confocal parameter $b$ of the pump beam must be adapted in order to keep the axial intensity about constant within the region explored by the probe beam. The data presented in Fig. 6 have been recorded in $\mathrm{O}_{2}$ using a longer focal length $(f=45 \mathrm{~cm}, b \approx 12.5 \mathrm{~mm})$, as compared to the experiment in $\mathrm{CO}_{2}(f=20 \mathrm{~cm}$, $b \approx 2.6 \mathrm{~mm}$ ), a magnification factor $M=-1.06$, and a probe beam diameter of $8 \mathrm{~mm}$. The FTOP image (top of the figure) displays six well resolved alignment transients recorded over a temporal window of $18 \mathrm{ps}$ (the rotational period of $\mathrm{O}_{2}$ is $T_{\mathrm{r}} \approx 11.6 \mathrm{ps}$ ). The permanent alignment is clearly identifiable. We should point out that despite the short acquisition time (only $100 \mathrm{~ms}$ ), the excellent resolution and signal to noise ratio makes the measurement fully exploitable for a detailed analysis of the alignment dynamics over a long time scale. The numerical simulation reported in the same figure has been obtained for a peak intensity $I_{0 \mathrm{p}}=49 \mathrm{TW} / \mathrm{cm}^{2}$ close to the experimental value $47 \mathrm{TW} / \mathrm{cm}^{2}$ estimated from the measure of the beam size $\left(\omega_{0} \approx 40 \mu \mathrm{m}\right)$. For linear molecules (or more generally for symmetric top molecules), the alignment transients produced at long time are solely affected by centrifugal distortion and collisions occurring in the gas sample. Both effects are included in the calculation. For indication, at atmospheric pressure and room temperature, the signal damping due to collisions is $15 \%$ at 18 ps. The curves shown with dashed lines indicate the spatial profile of the probe beam along the $y$ coordinates. The variation of the probe intensity is mainly responsible for the signal attenuation observed at the borders of the region recorded by the camera. The alignment of $\mathrm{O}_{2}$ in terms of efficiency is comparable to what observed in Fig. 5, with a $\left\langle\cos ^{2} \theta\right\rangle$ value (not shown) modulated between $0.54(\max )$ and $0.2(\mathrm{~min})$ at $I_{0 \mathrm{p}}=49 \mathrm{TW} / \mathrm{cm}^{2}$.

\section{CONCLUSION}

Snapshot imaging of postpulse alignment revival structures has been achieved using the single-shot FTOP tech- 
nique. The method employed for the characterization reveals many advantages with respect to preceding singleshot measurements. (i) Compared to Refs. [18, 20], the alignment can be produced over a very short interaction length which is more suitable for the use of large laser intensities needed for alignment. In fact the intensity can be increased much above the onset of ionization, since the birefringence signal is not affected by free electrons in a non-relativistic regime. On the contrary, the phase is very sensitive to electrons [28] which prevents spectral interferometry $[19,20]$ to be used at large intensity. (ii) The gas pressure in the static cell, although lower than the one used in Refs. [18, 19], can be further reduced. In our measurements, the sensitivity is rather limited by the additional depolarization of the probe field introduced by the windows of the gas cell than by the signal-to-noise ratio. This additional component can be reduced by introducing a uniform field component in phase quadrature with the probe field combined with an heterodyne detection. (iii) The temporal window for the detection can be adjusted as to cover the entire rotational period of most of the molecules. In the present work, the maximum range was limited to 18 ps, i.e. 9, 14, and 36 times the temporal domain reported in Refs. [19], [20], and [18], respectively. This value could be easily extent by use of a telescoped probe beam. (iv) The cross beam geometry used in the FTOP technique is well suited for measuring alignment produced by elliptically polarized laser pulses. Finally, we should add that the viability of the technique to infer the degree of alignment from the measurements is clearly demonstrated through the agreement obtained between the calculated and observed signals.

Several improvements to the method are envisaged. In the present setup, the signal recorded on each camera pixel results from an integration of the alignment produced along the propagation axis of the probe pulse. The measured signal results therefore from molecules exposed to different intensities of the laser field. This inconvenient can be obviated by using a microscopic objective for improving the depth resolution of the imaging system. Moreover, the technique can be extended to investigate molecules with low rotational temperature. This requires to integrate a part of the detection system to a supersonic free jet and to improve the detection by imaging two parallel planes, one of them containing the aligned molecules [29].

Fast recording of alignment revivals over a large temporal domain constitutes a major asset regarding optimal control of molecular alignment. Optimization using evolutionary algorithm in a closed feedback loop [30] is a time consuming process. The need to reduce the acquisition times is therefore crucial. Additionally, depending on the molecular system and the experimental conditions, the revival where the maximum degree of alignment can be achieved is not a priori known. The possibility of measuring simultaneously several transients can become necessary. The same applies if one wants to favor the alignment of a specific molecule present in a mixtures of different molecular species or isotopes, but also to control the alignment in dissipative media [31]. In this respect, the reported results represent a significant experimental advance in the field.

\section{Acknowledgments}

This work was supported by a Marie Curie European Reintegration Grant within the 6th European Community RTD Framework, the Conseil Régional de Bourgogne, and the CNRS.
[1] T. Seideman, Phys. Rev. Lett. 83, 4971 (1999).

[2] H. Stapelfeldt and T. Seideman, Rev. Mod. Phys. 75, 543 (2003).

[3] F. Rosca-Pruna and M. J. J. Vrakking, Phys. Rev. Lett. 87, 153902/1 (2001).

[4] V. Renard, M. Renard, S. Guérin, Y. T. Pashayan, B. Lavorel, O. Faucher, and H. R. Jauslin, Phys. Rev. Lett. 90, 153601 (2003).

[5] P. W. Dooley, I. V. Litvinyuk, K. F. Lee, D. M. Rayner, M. Spanner, D. M. Villeneuve, and P. B. Corkum, Phys. Rev. A 68, 23406 (2003).

[6] E. Hertz, D. Daems, S. Guérin, H. R. Jauslin, B. Lavorel, and O. Faucher, Phys. Rev. A 76, 043423 (2007).

[7] K. F. Lee, D. M. Villeneuve, P. B. Corkum, S. Albert, and G. U. Jonathan, Phys. Rev. Lett. 97, 173001 (2006).

[8] S. S. Viftrup, V. Kumarappan, S. Trippel, H. Stapelfeldt, E. Hamilton, and T. Seideman, Phys. Rev. Lett. 99, 143602 (2007).

[9] M. Leibscher, I. S. Averbukh, and H. Rabitz, Phys. Rev. Lett. 90, 213001 (2003).
[10] M. Leibscher, I. S. Averbukh, and H. Rabitz, Phys. Rev. A 69, 013402 (2004).

[11] E. Hertz, A. Rouzée, S. Guérin, B. Lavorel, and O. Faucher, Phys. Rev. A 75, 031403(R) (2007).

[12] A. Rouzé, E. Hertz, B. Lavorel, and O. Faucher, J. Phys. B 41, 074002 (2008).

[13] C. Z. Bisgaard, M. D. Poulsen, E. Peronne, S. S. Viftrup, and H. Stapelfeldt, Phys. Rev. Lett. 92, 173004 (2004).

[14] C. Z. Bisgaard, S. S. Viftrup, and H. Stapelfeldt, Phys. Rev. A 73, 053410 (2006).

[15] H. Stapelfeldt, Eur Phys J D 26, 15 (2003).

[16] A. Rouzée, S. Guérin, V. Boudon, B. Lavorel, and O. Faucher, Phys. Rev. A 73, 033418 (2006).

[17] V. Renard, O. Faucher, and B. Lavorel, Opt. Lett. 30, 70 (2005).

[18] S. Zamith, Z. Ansari, F. Lepine, and M. J. J. Vrakking, Opt. Lett. 30, 2326 (2005).

[19] Y. H. Chen, S. Varma, A. York, and H. M. Milchberg, Opt. Express 15, 11341 (2007).

[20] K. Hartinger and R. A. Bartels, Appl. Phys. Lett. 92, 
021126 (2008).

[21] M. Fujimoto, S. Aoshima, M. Hosoda, and Y. Tsuchiya, Phys. Rev. A 64, 033813 (2001).

[22] M. Fujimoto, S. Aoshima, M. Hosoda, and Y. Tsuchiya, Opt. Lett. 24, 850 (1999).

[23] M. Fujimoto, S. Aoshima, and Y. Tsuchiya, Meas. Sci. Technol. 13, 1698 (2002).

[24] J. G. Underwood, M. Spanner, M. Y. Ivanov, J. Mottershead, B. J. Sussman, and A. Stolow, Phys. Rev. Lett. 90, 223001/1 (2003).

[25] V. Renard, M. Renard, A. Rouzé, S. Guérin, H. R. Jauslin, B. Lavorel, and O. Faucher, Phys. Rev. A 70, 033420 (2004).

[26] B. J. Sussman, J. G. Underwood, R. Lausten, M. Ivanov, and A. Stolow, Phys. Rev. A 73, 53403 (2006).
[27] F. Rosca-Pruna and M. J. J. Vrakking, J. Chem. Phys. 116, 6579 (2002).

[28] A. Rouzée, V. Renard, S. Guérin, O. Faucher, and B. Lavorel, Phys. Rev. A 75, 013419 (2007).

[29] S. Minardi, A. Gopal, M. Tatarakis, A. Couairon, G. Tamošauskas, R. Piskarskas, A. Dubietis, and P. D. Trapani, Opt. Lett. 33, 86 (2008).

[30] C. Horn, M. Wollenhaupt, M. Krug, T. Baumert, R. de Nalda, and L. Banares, Phys. Rev. A 73, 31401(R) (2006).

[31] A. Pelzer, S. Ramakrishna, and T. Seideman, J. Chem. Phys. 126, 034503 (2007).

[32] V. Loriot, E. Hertz, B. Lavorel, and O. Faucher, in preparation. 